\title{
“VIEJO MUNDO” FRENTE A “NUEVO MUNDO” EN EL MERCADO BRITÁNICO DEL VINO. PERSPECTIVAS TRAS EL BREXIT
}

\author{
$\underline{\text { Raúl Compés }}^{*}$ y Francesc J. Cervera ${ }^{b *}$
} a Centro Internacional de Altos Estudios Agronómicos Mediterráneos (CIHEAM) (Zaragoza,
raul.compes@iamz.ciheam.org). b Universitat Politècnica de València (UPV) (València,
fracerif@ade.upv.es).

\section{Resumen}

El mercado británico es uno de los más importantes del mundo del vino. Además del elevado peso que tienen su consumo interior y sus importaciones en el total mundial, sigue siendo un mercado muy influyente y con elevados precios medios. En este contexto, la salida del Reino Unido de la Unión Europea va a modificar las relaciones comerciales con sus antiguos socios. Con toda probabilidad, las nuevas condiciones administrativas de acceso al mercado van a afectar negativamente a los países exportadores de vino de la Unión Europea (UE). Los más beneficiados podrían ser los países del "nuevo mundo".

Esta comunicación compara el comportamiento en el mercado británico de las exportaciones de vino del "viejo" y del "nuevo" mundo antes del Brexit, ya que podrían aportar información útil para prever el comportamiento futuro de las exportaciones de cada bloque. El resultado es que existen diferencias en materia de diversificación y competitividad entre ambos. En lo que respecta a la competitividad, el "viejo mundo" pierde frente al "nuevo" para el total de los vinos, aunque gana en las categorías de espumosos y vinos embotellados con DO. En el nuevo marco post Brexit, los exportadores de la UE deberían concentrar sus estrategias en estas categorías.

Palabras clave: Brexit, vino, viejo mundo, nuevo mundo, competitividad.

\section{Introducción}

El Reino Unido es uno de los mercados con mayor influencia en el comercio internacional de vino. Esto viene ocurriendo desde el siglo XIX (Ludington, 2013), y se mantiene gracias al tamaño de su mercado, su peso en el negocio de la distribución y el gran poder de irradiación del mundo anglosajón. En este contexto, la salida del Reino Unido de la Unión Europea mediante una fórmula de "Brexit duro" va a suponer un aumento de los obstáculos al comercio con los países comunitarios [véase Bonadio y Mimler (2019); Duarte y Kok (2019)]. Según Anderson y Wittwer (2017), el Brexit va a tener efectos negativos, entre ellos un aumento del precio del vino, lo que podría generar un efecto disuasorio en su consumo y una reducción de las importaciones. Todo ello repercutiría negativamente en los exportadores de vino, algunos muy dependientes del mercado británico.

El mercado británico es muy dinámico, y en los últimos años se han producido cambios estructurales que pueden anticipar la situación post Brexit [véase Compés y Cervera (2019)b]. El modelo tradicional, basado mayoritariamente en importaciones de vino embotellado procedente de la UE, ha dado paso a un modelo mixto compuesto, por un lado, por importaciones de vino barato a granel -en gran parte procedente de países del "nuevo" mundo- y, por otro, de vino embotellado de mayor precio, procedente del "viejo mundo" europeo.

Este cambio ha ido acompañado del surgimiento de una actividad exportadora, constituida tanto por operaciones puras de reexportación de vino a granel con ventas exteriores de vino embotellado en el Reino Unido. Estos cambios obedecen a distintas causas, pero dos de ellas son el paulatino surgimiento de una industria vinícola empujada por los efectos positivos del cambio climático en algunas partes de la isla y por la tendencia a evitar el transporte de vino embotellado para reducir las emisiones de gases efecto invernadero, sobre todo en las gamas bajas.

En el nuevo escenario tras el Brexit es previsible que este modelo se polarice, lo que consolidaría un segmento de importaciones de vinos baratos a granel procedentes de países del "nuevo mundo" y otro de vinos con Denominación de Origen procedentes de la UE. Este escenario es consistente con establecer acuerdos de libre comercio con aquellos países proveedores del "nuevo mundo" con los que todavía existen tienen barreras comerciales y, a pesar del acuerdo comercial para no imponer aranceles ni cuotas entre el Reino Unido y la Unión Europea, establecer requisitos documentales, controles e inspecciones de a los vinos embotellados procedentes de la UE.

\section{Objetivos}

El objetivo principal de esta comunicación es determinar el papel del Reino Unido en los mercados mundiales de vino y, a partir de ahí, prever las consecuencias de un aumento de los obstáculos comerciales aplicados a los vinos procedentes de la Unión Europea, que a priori van a ser los perdedores de las nuevas barreras comerciales británicas. 
Para ello se analizan dos indicadores de desempeño de las importaciones de vino del Reino Unido con origen en los principales exportadores a nivel mundial, comparando la situación entre las procedentes del "viejo mundo" y las del "nuevo mundo". El primer indicador evalúa el grado de diversificación por tipo de vino, su objetivo es determinar el grado de especialización por producto de los principales proveedores del mercado británico. El segundo indicador evalúa la competitividad de estos mismos proveedores en cada tipo y categoría de producto con el fin de determinar su evolución en el mercado británico. La utilización conjunta de estos indicadores permite determinar el desempeño de las exportaciones de ambos bloques.

\section{Metodología}

La metodología utilizada es la aplicada por Cervera y Compés (2018) para estudiar la competitividad en los mercados internacionales del vino. La agregación de países del "viejo mundo" comprende a España, Francia, Italia, Alemania y Portugal y, para el "nuevo mundo", a EEUU, Chile, Argentina, Sudáfrica, Australia y Nueva Zelanda).

Se han estudiado tres tipos de productos, en valor: espumosos (220410), embotellados (220421) y a granel (220429, incluyendo esta categoría la partida 220422 -Bag in Box-aplicada a partir del 01/01/2017). Adicionalmente se han seleccionado cuatro calidades ${ }^{17}$ : vinos con Denominación de Origen (DO), vinos con Indicación Geográfica Protegida (IGP), vinos Varietales, y otros vinos.

Para analizar la diversificación por productos se ha utilizado el Índice de Diversificación [véase Mimouni et al. (2007)] y para el análisis de la competitividad por mercados se ha utilizado en Índice de Competitividad Global [véase COGEA (2014)].

\section{Resultados}

\subsection{Diversificación por productos}

Las exportaciones del "viejo mundo" vitivinícola se han ido concentrando hacia vinos embotellados de calidad (espumosos y tranquilos con DO), en detrimento de vinos tranquilos con IGP y otros vinos -sin calidad certificada ni varietales-, lo que se refleja en que su índice de diversificación haya pasado de 3,91 en 2010 a 3,41 en 2019.

Por el otro lado, las del "nuevo mundo" han reducido su concentración por categorías de vino, debido a la caída del peso de los vinos embotellados sin calidad certificada ni varietales y al aumento del peso de los vinos embotellados varietales y, con mayor intensidad, de los graneles sin calidad certificada ni varietales. Esto se manifiesta en un aumento del índice de diversificación, que ha pasado de 2,06 en 2010, a 3 en 2019.

\subsection{Competitividad}

La competitividad de los vinos del "viejo mundo" vitivinícola, en su conjunto, en el mercado británico durante la década del 2010 y 2020 ha decrecido significativamente, con unas pendientes de la regresión logarítmica para la variación de las exportaciones y variación de la cuota de mercado negativas, de -1,388 y - 0,022 , respectivamente. Por su parte, el conjunto del "nuevo mundo" vitivinícola ha ganado significativamente competitividad, con ambas pendientes de la regresión logarítmica positivas, de 0,98, para la variación de las exportaciones, y 0,02, para la variación de la cuota de mercado (Cuadro 1).

Por tipos de producto, el "viejo mundo" vitivinícola obtiene un aumento significativo de la competitividad de sus exportaciones de vinos espumosos, mientras decrece levemente para los vinos embotellados y significativamente en los vinos a granel. Para el "nuevo mundo" ocurre exactamente contrario; sus graneles ganan competitividad de forma significativa, mientras que sus embotellados lo hacen moderadamente y, en el caso de los espumosos, decrece de forma significativa.

Por calidades de producto se observa que, en primer lugar, crece moderadamente la competitividad de los vinos con Denominación de Origen del "viejo mundo" mientras que se reduce la de los del "nuevo mundo". En segundo lugar, la competitividad de los vinos con Indicación Geográfica Protegida del viejo mundo se reduce levemente, mientras que crece moderadamente la de los del "nuevo mundo". En tercer lugar, se observa un decrecimiento significativo de la competitividad de los vinos varietales del "viejo mundo" mientras que la de los del "nuevo mundo" crece significativamente. Por último, se reduce la competitividad de los vinos del "viejo mundo" que no tienen calidad certificada ni son varietales y, por el contrario, aumenta notablemente la de los del "nuevo mundo".

\footnotetext{
${ }^{17}$ Esta división se ha realizado a nivel de subpartidas arancelarias, gracias a las definiciones del portal Wine Trade Data de la UE -EC - DG AGRI - Wine Trade Data (europa.eu)-.
} 
Cuadro 1. Análisis del Índice de Competitividad Global en los mercados individuales de categoría de producto de las exportaciones de vino del vino del viejo mundo vitivinícola (VM) y del nuevo mundo vitivinícola (NM) en el mercado del Reino Unido entre el 2010 y el 2019

\begin{tabular}{|c|c|c|c|c|c|c|}
\hline \multirow{2}{*}{$\begin{array}{l}\text { Categoría } \\
\text { de vino }\end{array}$} & \multicolumn{2}{|c|}{$\begin{array}{l}\text { Variación de las } \\
\text { Exportaciones }\end{array}$} & \multicolumn{2}{|c|}{$\begin{array}{c}\text { Variación de la } \\
\text { Cuota de Mercado }\end{array}$} & \multicolumn{2}{|c|}{ Evolución de la competitividad } \\
\hline & VM & NM & VM & NM & VM & NM \\
\hline TOTAL & $-1,388$ & 0,979 & $-0,022$ & 0,020 & $\begin{array}{c}\text { Decrece } \\
\text { significativamente }\end{array}$ & $\begin{array}{c}\text { Crece } \\
\text { significativamente }\end{array}$ \\
\hline Espumosos & 18,975 & $-20,479$ & 0,034 & $-0,043$ & $\begin{array}{c}\text { Crece } \\
\text { significativamente }\end{array}$ & $\begin{array}{c}\text { Decrece } \\
\text { significativamente }\end{array}$ \\
\hline Embotellados & $-0,058$ & 0,004 & 0,016 & $-0,021$ & Decrece levemente & Crece levemente \\
\hline Granel & $-60,440$ & 59,063 & $-0,132$ & 0,131 & $\begin{array}{c}\text { Decrece } \\
\text { significativamente }\end{array}$ & $\begin{array}{c}\text { Crece } \\
\text { significativamente }\end{array}$ \\
\hline DO & 6,336 & $-7,750$ & $-0,003$ & $-0,004$ & Crece levemente & $\begin{array}{c}\text { Decrece } \\
\text { significativamente }\end{array}$ \\
\hline IGP & $-14,899$ & 15,645 & 0,010 & $-0,022$ & Decrece levemente & Crece levemente \\
\hline Varietal & $-27,152$ & 23,746 & $-0,196$ & 0,191 & $\begin{array}{c}\text { Decrece } \\
\text { significativamente }\end{array}$ & $\begin{array}{c}\text { Crece } \\
\text { significativamente }\end{array}$ \\
\hline Otros & $-0,069$ & 0,959 & $-0,008$ & 0,016 & $\begin{array}{c}\text { Decrece } \\
\text { significativamente }\end{array}$ & $\begin{array}{c}\text { Crece } \\
\text { significativamente }\end{array}$ \\
\hline
\end{tabular}

Elaboración propia con datos de DATACOMEX

\section{Conclusiones}

En el siglo XXI, en el comercio exterior británico del vino se han producido cambios estructurales significativos. Por un lado, sus importaciones totales han perdido peso en el mercado mundial, se ha reducido su precio unitario y ha aumentado la participación de los vinos procedentes del "nuevo mundo". Por otro lado, han crecido las exportaciones de vino elaborado a partir de estas importaciones, con destino principalmente a la UE. Se trata, por tanto, de una tendencia que indica una dualización caracterizada por la importación de más vinos a granel procedentes del nuevo mundo y menos vinos embotellados procedentes del viejo mundo. En parte como consecuencia de este cambio, ha aparecido una industria propia con vocación exportadora.

El análisis elaborado en esta comunicación, a partir de indicadores de diversificación y competitividad no sólo corrobora estas tendencias sino que permite entender mejor el nuevo modelo de abastecimiento de la demanda británica de vino. El "viejo mundo" aumenta su competitividad en vinos embotellados con DO en los que ha aumentado su especialización- y el "viejo mundo" lo hace en vinos a granel, varietales y con IGP -en el que también se ha especializado-. Este nuevo modelo puede verse acentuado en la nueva etapa tras el Brexit, que para los exportadores de la UE va a significar más trámites y trabas aduaneras, lo que obliga a los exportadores europeos a concentrarse en los vinos con mayor calidad, cuyo alto precio permite soportar mejor -en términos relativos- estos nuevos costes.

\section{Bibliografía}

Anderson K. y Wittwer, G. (2017). The UK and Global Wine Markets by 2025, and Implications of Brexit. Wine Economics Research Centre. Journal of Wine Economics, 12(3):221-251.

Bonadio, E., y Mimler, M. (2019). "Brexit Implications for Geographical Indications of Food and Beverages". European Intellectual Property Review, 1.

Cervera, F.J. y Compés, R. (2018). "The performance of Spanish wine exports in international markets". Economía Agraria y Recursos Naturales - Agricultural and Resource Economics, 18(1):23-48.

COGEA. (2014). Study on the competitiveness of European wines. Office for Official Publications of the European Communities. Luxemburg.

Compés, R. y Cervera, F.J. (2019)a. "The commercial performance factors of the large exporting countries in bottled and bulk wine markets in the 21 st century". BIO Web of Conferences, 12:3008.

Compés, R. y Cervera, F.J. (2019)b. "El comercio exterior de vino del Reino Unido ante el Brexit". XII Congreso de Economía Agraria, 4-6/09, Lugo (España).

Duarte, A., y Kok, S. (2019). "Dynamic capabilities in the context of Brexit and international wine business: An exploratory two-country study". Thunderbird International Business Review, 61(2):277-290.

Ludington, C. (2013). The Politics of Wine in Britain. Palgrave Macmillan, Londres (Reino Unido). 
Mimouni, M., Fontagné, L. y Von Kirchbach, F. (2007). The Trade Performance Index. Technical Notes. Market Analysis Section-International Trade Centre. Ginebra (Suiza). 